\title{
Comment on "Experimental and theoretical study of the triple-differential cross section for electron-impact ionization of thymine molecules"
}

\author{
S. Houamer, ${ }^{1, *}$ C. Dal Cappello, ${ }^{2}$ I. Charpentier, ${ }^{3}$ P. A. Hervieux, ${ }^{4}$ and A. C. Roy ${ }^{5}$ \\ ${ }^{1}$ Laboratoire de Physique Quantique et Systèmes Dynamiques, Département de Physique, Faculté des Sciences Université Ferhat Abbas, \\ Sétif 19000, Algeria \\ ${ }^{2}$ Laboratoire Structure et Réactivité des systèmes moléculaires UMR 7565, Université de Lorraine, 1 Boulevard Arago, \\ 57078 Metz Cedex 3, France \\ ${ }^{3}$ Laboratoire d'Etudes des Microstructures et de Mécanique des Matériaux, UMR 7239, Université de Lorraine, Ile du Saulcy, \\ 57045 Metz Cedex 1, France \\ ${ }^{4}$ Institut de Physique et Chimie des Matériaux de Strasbourg, Université de Strasbourg, 23 rue du Loess, Boîte Postale 43, \\ 67034 Strasbourg Cedex 2, France \\ ${ }^{5}$ School of Mathematical Sciences, Ramakrishna Mission Vivekananda University, Belur Math 711202, West Bengal, India
}

(Received 11 April 2012; published 22 August 2012)

\begin{abstract}
In their recent paper, Bellm et al. [Phys. Rev. A 85, 022710 (2012)] performed (e,2e) experiments on thymine at an incident energy of $250 \mathrm{eV}$. They wrote in the conclusion that a model based on the first Born approximation using the completely neglected differential overlap description is in very good agreement with the experimental data. On the contrary, we argue that this model fails to describe experiments on water performed at the same incident energy and is unable to explain any shift of the binary or recoil peaks.
\end{abstract}

DOI: 10.1103/PhysRevA.86.026701

PACS number(s): $34.80 . \mathrm{Gs}$

The ionization of molecules of biological importance is a fundamental step to understand the interaction of charged particles with the human body. The $(e, 2 e)$ experiments, which detect the scattered and the ejected electrons in time coincidence, are an important tool to investigate the tripledifferential cross section (TDCS) which provides a stringent test of an ionization process.

Bellm et al. [1] performed difficult $(e, 2 e)$ experiments on inner valence orbitals of thymine at an incident energy of $250 \mathrm{eV}$ and an ejected-electron energy of $20 \mathrm{eV}$. The difficulties are due to the measurements of inner valence orbitals of thymine which are very closely spaced in energy. Therefore, it is difficult to separate each molecular orbital of thymine. Presumably, their measurements contain contributions from eight molecular orbitals. Moreover, we notice that there are two conformers of the thymine molecule with small differences in orbital ionization between these two structures [2]. Thus one may assume that these differences are small for the molecular wave functions too. It is well worth noting that the experiment of Bellm et al. [1] suffers from one drawback: all the measured data are relative.

In the section of theoretical framework Bellm et al. [1] write that in their work on the $(e, 2 e)$ experiment with coplanar asymmetric kinematics, the Coulomb Born approximation, also referred to as the first Born approximation (FBA), is completely justified. It is not quite true, because we notice that at the scattering angle of $10^{\circ}$ (see Fig. 3 of Ref. [1]) the TDCS shows a single binary lobe centered close to the momentum transfer direction with a small shift of approximately $5^{\circ}$ to larger ejected-electron angles. As the TDCS provides the most detailed information of an ionization process, it is impossible for a simple method like FBA to reproduce all the features of an $(e, 2 e)$ experiment. For example, Brauner et al. [3] introduced a model, usually referred to as the Brauner-Briggs-Klar (BBK)

*Corresponding author: s_houamer@univ-setif.dz model, to calculate the TDCS for the ionization of atomic hydrogen at a high incident energy of $250 \mathrm{eV}$ (which is nearly 18 times the ionization threshold) and for a fixed ejectedelectron energy of $5 \mathrm{eV}$ in a coplanar geometry, and compared their results with the corresponding absolute measurements of Ehrhardt et al. [4] and FBA calculations. They found that FBA failed to reproduce the shifts in the binary and recoil peaks observed in the experiment. The reason is that $F B A$ cross sections are symmetric about the direction of momentum transfer. It is a result which is well known and was also a result found by other authors using the second Born approximation (SBA) [5] which is more successful than the FBA. When the energy of the ejected electron increases while the incident energy is kept fixed, i.e., for symmetric or near symmetric geometry, FBA fails as the SBA [6,7]. In that geometry only the BBK model or more sophisticated theories such as continuumdistorted-wave-eikonal-initial-state [8,9], convergent closecoupling $[10,11]$, or any model which includes the interaction between the scattered and ejected electrons are able to give good agreement. As a matter of fact, FBA becomes exact in the limit of infinite energy. Furthermore, Brauner et al. [3] find that even for an incident energy of $2000 \mathrm{eV}$ a difference of a few percent exists between the results of BBK and FBA methods.

Figure 1 shows that the FBA using the completely neglected differential overlap (CNDO) model is clearly insufficient to describe the experimental data. A better description is provided by the DS3C model (together with the CNDO approximation) which uses the BBK model with effective charges [12,13]. The BBK model which includes Coulomb interactions in pairs among the three charged particles in the final state also yields a TDCS in good agreement with the experimental data of Bellm et al. [1]. We would like to remark that the present FBA results are the same as those in Ref. [14]. Such a shift of the binary peak is also present in both the experiments of Colyer et al. [15] on the ionization of tetrahydrofuran and the experiments of Builth-Williams et al. [16] on the ionization of pyrimidine 


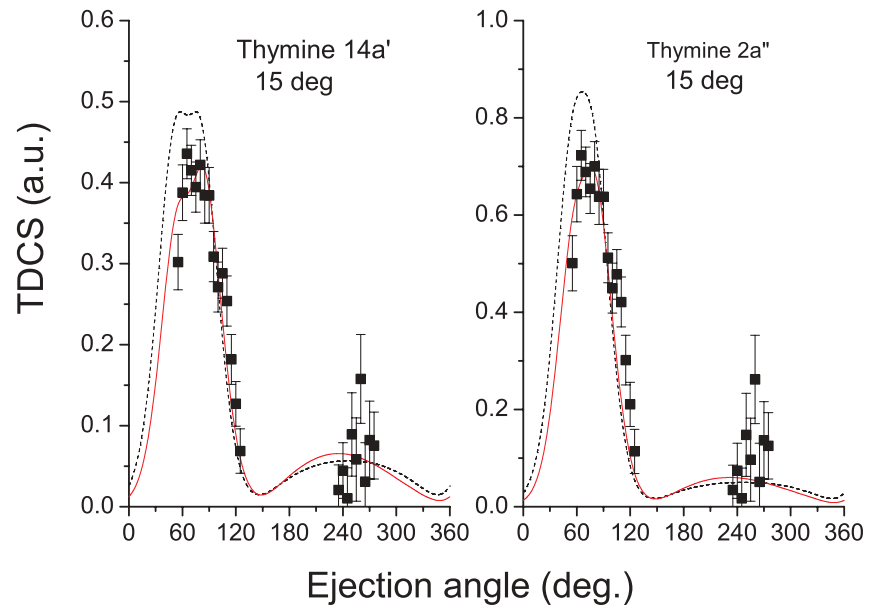

FIG. 1. (Color online) Triple-differential cross section for $250 \mathrm{eV}$ electron-impact ionization of the $2 a^{\prime \prime}$ and $14 a^{\prime}$ orbitals of thymine. The theoretical calculations are performed using the FBA (dashed line) and the DS3C methods (solid line). Both methods use the CNDO approximation. The energy of the ejected electron $E_{e}$ is $20 \mathrm{eV}$, while the scattered angle is fixed at $15^{\circ}$. Solid rectangles represent the experimental data.

molecules. One notices that the M3DW model [17], used to explain these two experiments $[15,16]$, is able to reproduce a part of the shift because this model, like the BBK and the DS3C models, takes into account the Coulomb repulsion between the two emitting electrons in the final state.

Next we would like to elaborate on the discussion of the CNDO model used by Bellm et al. [1]. This model, developed within the FBA framework, considers the TDCS as a weighted sum of atomic triply differential cross sections corresponding to the different atomic components involved in the molecular subshell [18]. Originally, this model was developed for the study of the doubly differential cross sections corresponding to the ionization of molecules by protons [18] and gave a reasonably good agreement with experiments. While applying this CNDO model the authors did not take into account the molecular wave function directly as was done, for instance, in the study of the ionization of water by electrons $[19,20]$. (In these two papers the Moccia wave function was used to describe the initial molecular state.) Instead, they used input parameters for the occupied molecular orbitals obtained from an $a b$ initio method using the GAUSSIAN09 software. Such a great (and interesting) simplification has never been checked so far in the calculations of TDCS. Thus we decided to check this model in the case of ionization of the water molecule for which $(e, 2 e)$ experiments are available with the same kinematics $(250 \mathrm{eV}$ for the incident electron). We apply the following populations for the water molecular orbitals [21]:

$$
\begin{aligned}
& 0.50 \mathrm{H}(1 s)+1.50 \mathrm{O}(2 s) \\
& 2 \mathrm{O}(2 s) \\
& 0.34 \mathrm{H}(1 s)+0.20 \mathrm{O}(2 s)+1.46 \mathrm{O}(2 p) \\
& 0.82 \mathrm{H}(1 s)+1.18 \mathrm{O}(2 p)
\end{aligned}
$$

Figure 2 shows a comparison between the experiments of Milne-Brownlie et al. [19] and the CNDO model developed by Bellm et al. [1]. We notice good agreement for the ionization of

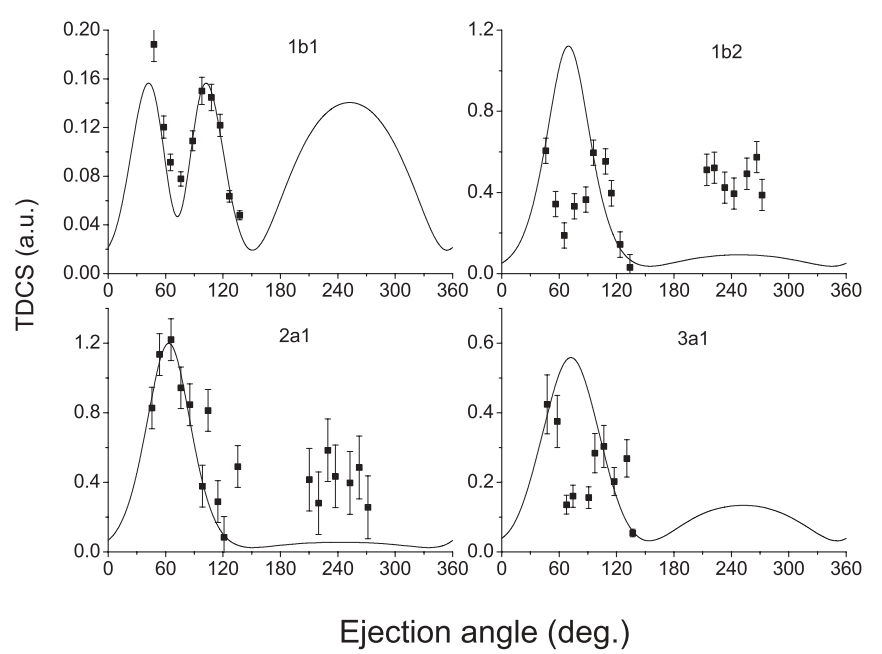

FIG. 2. Triple-differential cross section for $250 \mathrm{eV}$ electronimpact ionization of the valence orbitals of water $\left(1 b_{1}, 1 b_{2}, 2 a_{1}\right.$, and $3 a_{1}$ ). The theoretical calculations are performed in the FBA (solid line). The energy of the ejected electron is $8 \mathrm{eV}$ for $3 a 1$ and $10 \mathrm{eV}$ for the other orbitals, while the scattering angle is fixed at $15^{\circ}$. Solid rectangles represent the experimental data.

the $2 a_{1}$ and $1 b_{1}$ orbitals and full disagreement for the ionization of $3 a_{1}$ and $1 b_{2}$ orbitals. We recall that the FBA used in MilneBrownlie et al. [19] or in Champion et al. [20] gives relative good agreement for the ionization of any water molecular orbital (except the shift observed for the ionization of $2 a_{1}$ ). This means that the use of a molecular wave function is more appropriate for calculating the TDCS than a weighted sum of atomic triply differential cross sections corresponding to the different atomic components.

A second check consists of comparing the results given by the FBA with the CNDO approach with the well-known electron momentum spectroscopy (EMS) experiments. We remind that EMS experiments are performed at high impact energy where the FBA is valid and we investigate the case of methane.

We have the following populations for the methane molecular orbitals [21] while applying the CNDO model:

$$
\begin{aligned}
& 0.25 \mathrm{C}(2 s)+0.75 \mathrm{C}(2 p)+1 \mathrm{H}(1 s) \\
& 0.75 \mathrm{C}(2 s)+2.25 \mathrm{C}(2 p)+3 \mathrm{H}(1 \mathrm{~s})
\end{aligned}
$$

Figure 3 shows that good agreement is found for the $2 a_{1}$ orbital but we observe full disagreement for the $1 t_{2}$ orbital when we compare CNDO results to the experiments of Clark et al. [22]. When a simple FBA model using the Moccia molecular wave function is used, good agreement with experiments is observed in both cases.

As a last check we study the TDCS for each of the thymine molecular orbitals by using the CNDO model proposed by Bellm et al. [1] (with the coefficients given in Table I [1]). Figure 4 shows the results for four molecular orbitals for a scattered electron of $10^{\circ}$. We observe the same shape for the $6 a^{\prime}, 14 a^{\prime}, 2 a^{\prime \prime}$, and $6 a^{\prime \prime}$. Interestingly, we also obtain the same shape for any valence molecular orbital presented in Table I of Bellm et al. [1]. We believe that this result is very surprising because the molecular orbitals are different and should give different results and different shapes [23] (as in the 


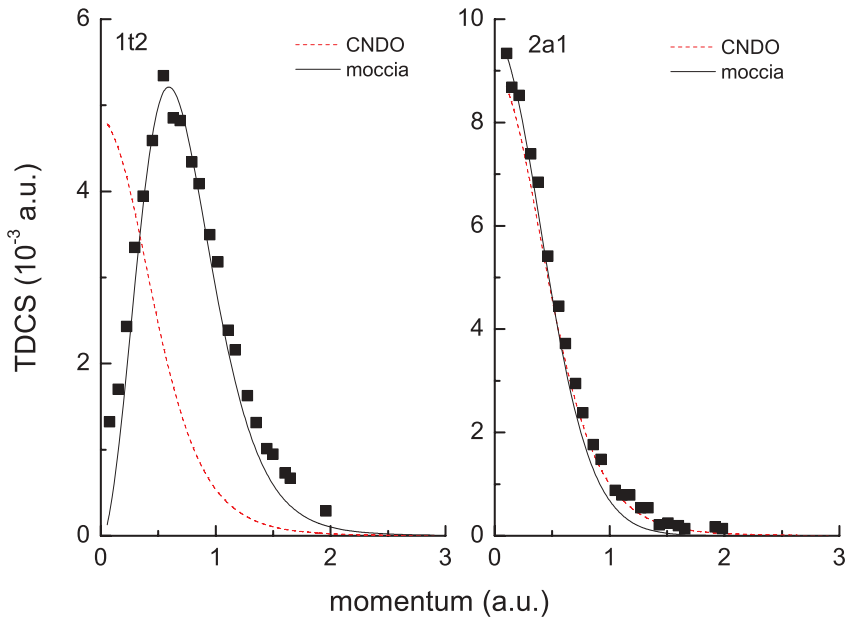

FIG. 3. (Color online) Comparison between the FBA in the case of the ionization of methane with the Moccia wave function (solid line), the FBA with the CNDO approximation (dashed line), and the experimental electron momentum density profiles (squares) from Ref. [22], obtained in a noncoplanar symmetric geometry at an incident energy $\sim 1200 \mathrm{eV}$. The absolute scale shown is that of the FBA calculations with the Moccia wave function.

case of the ionization of the water molecule, in the case of the methane molecule, or in the case of other biomolecules such as the pyrimidine molecule [16]). Furthermore, we have studied the other case $\left(15^{\circ}\right.$ for the scattered electron). In this case we obtain two possible shapes only, as those given by the $14 a^{\prime}$ and the $2 a^{\prime \prime}$ molecular orbitals. Here we have also investigated all valence molecular orbitals and have found no change for the shapes.

It has been indicated earlier that cross-section calculations for a particular orientation of the molecule exhibit large differences in shape and magnitude when compared to those after averaging [24]. The CNDO model cannot reproduce

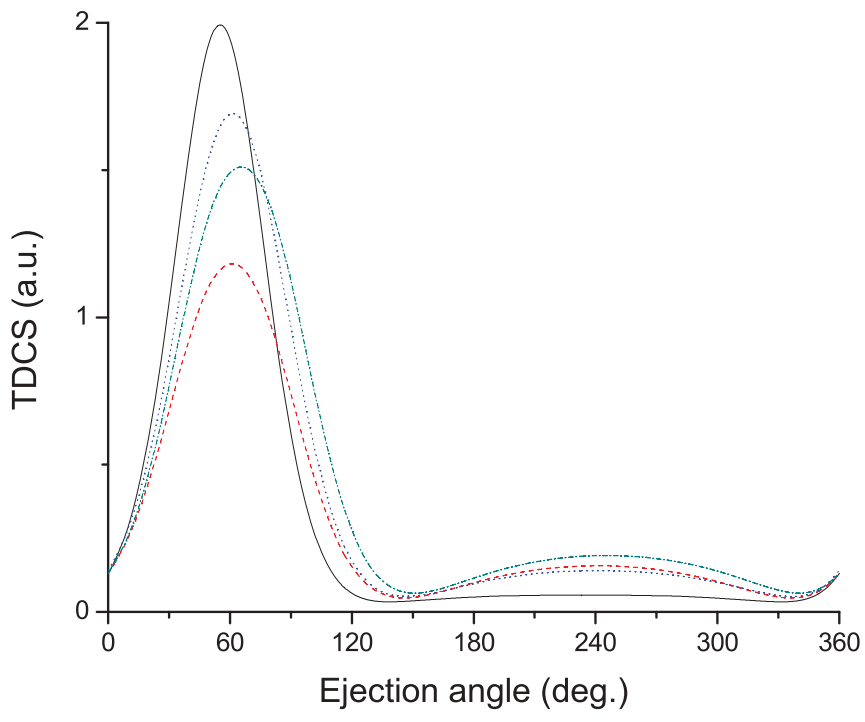

FIG. 4. (Color online) Triple-differential cross section for $250 \mathrm{eV}$ electron-impact ionization of thymine. The theoretical calculations are performed in the FBA: the solid line is the TDCS for the ionization of the $6 a^{\prime}$ inner valence orbital, the dashed line is the $14 a^{\prime}$ inner valence orbital, the dotted line is the $2 a^{\prime \prime}$ inner valence orbital, and the dash-dotted line represents the $6 a^{\prime \prime}$ valence orbital. The energy of the ejected electron is $20 \mathrm{eV}$ and the scattered angle is $10^{\circ}$.

the predictions of these kinds of calculations at all because the molecular wave function is not taken into account directly in the evaluation of TDCS.

Finally we conclude that the CNDO atomiclike model proposed by Bellm et al. [1] is not able to reproduce the experiments on the ionization of water or of methane and the agreement found for the ionization of thymine is somewhat questionable and fortuitous. We argue that a more sophisticated collision model going beyond the FBA and including the full molecular character of the orbitals is needed to explain all the details of $(e, 2 e)$ experiments.
[1] S. M. Bellm, C. J. Colyer, B. Lohmann, and C. Champion, Phys. Rev. A 85, 022710 (2012).

[2] O. Dolgounitcheva, V. G. Zakrzewski, and J. V. Ortiz, J. Phys. Chem. A 106, 305 (2006).

[3] M. Brauner, J. S. Briggs, and H. Klar, J. Phys. B 22, 2265 (1989).

[4] H. Ehrhardt, G. Knoth, P. Schlemmer, and K. Jung, Phys. Lett. A 110, 92 (1985); H. Ehrhardt, K. Jung, G. Knoth, and P. Schlemmer, Z. Phys. D 1, 3 (1986).

[5] F. W. Byron, Jr., C. J. Joachain, and B. Piraux, J. Phys. B 13, L673 (1980); Phys. Lett. A 99, 427 (1983); 106, 299 (1984); J. Phys. B 18, 3203 (1985).

[6] E. Weigold, C. J. Noble, S. T. Hood, and I. Fuss, J. Phys. B 12, 291 (1979).

[7] C. Dal Cappello, A. Haddadou, F. Menas, and A. C. Roy, J. Phys. B 44, 015204 (2011).

[8] D. S. F. Crothers and J. F. Mc Cann, J. Phys. B 16, 3229 (1983).

[9] S. Jones and D. H. Madison, Phys. Rev. Lett. 81, 2886 (1998).
[10] I. Bray, D. A. Konovalov, I. E. Mc Carthy, and A. T. Stelbovics, Phys. Rev. A 50, R2818 (1994).

[11] S. Jones and D. H. Madison, Phys. Rev. A 62, 042701 (2000).

[12] J. Berakdar and J. S. Briggs, Phys. Rev. Lett. 72, 3799 (1994).

[13] S. Zhang, J. Phys. B 33, 3545 (2000).

[14] S. M. Bellm, C. J. Colyer, B. Lohmann, and C. Champion, Phys. Rev. A 85, 039905(E) (2012).

[15] C. J. Colyer, S. M. Bellm, B. Lohmann, G. F. Hanne, O. Al-Hagan, D. H. Madison, and C. G. Ning, J. Chem. Phys. 133, 124302 (2010).

[16] J. D. Builth-Williams, S. M. Bellm, D. B. Jones, Hari Chaluvadi, D. H. Madison, C. G. Ning, B. Lohmann, and M. J. Brunger, J. Chem. Phys. 136, 024304 (2012).

[17] J. Gao, D. H. Madison, and J. L. Peacher, J. Chem. Phys. 123, 204314 (2005); Phys. Rev. A 72, 032721 (2005); J. Chem. Phys. 123, 204302 (2005). 
[18] B. Senger and R. V. Rechenmann, Nucl. Instrum. Methods Phys. Res. B 2, 204 (1984); B. Senger, Z. Phys. D 9, 79 (1988).

[19] D. S. Milne-Brownlie, S. J. Cavanagh, B. Lohmann, C. Champion, P.-A. Hervieux, and J. Hanssen, Phys. Rev. A 69, 032701 (2004).

[20] C. Champion, C. Dal Cappello, S. Houamer, and A. Mansouri, Phys. Rev. A 73, 012717 (2006).

[21] K. Siegbahn, C. Nordling, G. Johansson, J. Hedman, P. F. Heden, K. Hamrin, U. Gelius, T. Bergmark, L. O. Werne, R. Manne, and Y. Baer, ESCA Applied to Free Molecules (North Holland, Amsterdam, 1969).

[22] S. A. C. Clark, T. J. Reddish, C. E. Brion, E. R. Davidson, and R. F. Frey, Chem. Phys. 143, 1 (1990).

[23] C. Dal Cappello, Z. Rezkallah, S. Houamer, I. Charpentier, P. A. Hervieux, M. F. Ruiz-Lopez, R. Dey, and A. C. Roy, Phys. Rev. A 84, 032711 (2011).

[24] C. Champion, J. Hanssen, and P. A. Hervieux, Phys. Rev. A 63, 052720 (2001). 